\title{
Mobile-assisted ad hoc networking architecture based on location information
}

\author{
Shota Ono ${ }^{1 \text { a) }}$, Taku Yamazaki ${ }^{2}$, Takumi Miyoshi ${ }^{2,1}$, \\ and Kaoru Sezaki ${ }^{1}$ \\ ${ }^{1}$ Institute of Industrial Science, University of Tokyo, \\ 4-6-1 Komaba, Meguro-ku, Tokyo 153-8505, Japan \\ ${ }^{2}$ College of Systems Engineering and Science, Shibaura Institute of Technology, \\ 307 Fukasaku, Minuma-ku, Saitama-shi, Saitama 337-8570, Japan \\ a)shota@mcl.iis.u-tokyo.ac.jp
}

\begin{abstract}
This letter proposes a mobile-assisted ad hoc networking architecture based on location information. The proposed architecture comprises the location layer and ad hoc layer. The location layer performs to manage the locations of nodes and to determine the area of ad hoc network virtually based on locations via mobile networks. The ad hoc layer performs to establish an actual route based on the virtual area and to send data along the established route via local networks. The proposed architecture achieves the significant reduction of the unnecessary packets and the improvement of the packet arrival rate.
\end{abstract}

Keywords: ad hoc network, location information, mobile network, deviceto-device communication, relay range

Classification: Network System

\section{References}

[1] F. Jameel, Z. Hamid, F. Jabeen, S. Zeadally, and M. A. Javed, "A survey of device-to-device communications: Research issues and challenges," IEEE Commun. Surveys Tuts., vol. 20, no. 3, pp. 2133-2168, Apr. 2018. DOI:10. 1109/COMST.2018.2828120

[2] M. S. Corson, R. Laroia, J. Li, V. Park, T. Richardson, and G. Tsirtsis, "Toward proximity-aware internetworking," IEEE Wireless Commun., vol. 17, no. 6, pp. 26-33, Dec. 2010. DOI:10.1109/MWC.2010.5675775

[3] A. Boukerche, B. Turgut, N. Aydin, M. Z. Ahmad, L. Bölöni, and D. Turgut, "Routing protocols in ad hoc networks: A survey," Comput. Netw., vol. 55, no. 13, pp. 3032-3080, Sept. 2011. DOI:10.1016/j.comnet.2011.05.010

[4] B. A. Mahmood and D. Manivannan, "Position based and hybrid routing protocols for mobile ad hoc networks: A survey," Wireless Pers. Commun., vol. 83, no. 2, pp. 1009-1033, July 2015. DOI:10.1007/s11277-015-2437-8

[5] T. Miyoshi, Y. Shimomura, and O. Fourmaux, "A P2P-based communication framework for geo-location oriented networks," J. Telecommun. Inform. Tech. (JTIT), vol. 2019, no. 1, pp. 58-66, Mar. 2019. DOI:10.26636/JTIT.2019. 131119

[6] C. E. Perkins and E. M. Royer, "Ad-hoc on-demand distance vector routing," 2nd IEEE Workshop on Mobile Comput. Syst. and Applications (WMCSA 
1999), New Orleans, USA, pp. 90-100, Feb. 1999. DOI:10.1109/MCSA.1999. 749281

[7] NS-3, https://www.nsnam.org.

\section{Introduction}

Device to device (D2D) that realizes direct information exchange between neighbor devices is expected as a promising technology of wireless inter-node communication. Mobile network carriers attempt to introduce their own D2D services assisted by carriers' base stations [1]. Communication domain in such the carrier-assisted D2D services is limited due to the centralized system [2].

On the other hand, ad hoc network is able to realize D2D without any dependence on network carriers [3]. Ad hoc network constructs a multihop network distributedly and autonomously only via wireless communication between nodes; The ad-hoc-based D2D services do not rely on the mobile network carries. One of disadvantages on ad hoc network is to send many control messages in the routing process and then to consume excessive communication resources. To solve this issue, location-based routing methods have been proposed. However, location information of nodes is exchanged in a multihop manner and might be hardly enough diffused [4]. In addition, the location information becomes obsolete due to the high mobility of nodes. As a result, an appropriate communication route between the source and destination nodes cannot be constructed.

This letter proposes a mobile-assisted ad hoc network architecture in which the control messages are transferred only in a proper area. The method relies on the reliable location information, which is provided and maintained by mobile network.

\section{Mobile-assisted ad hoc networking architecture}

\subsection{Overview of the proposed architecture}

The proposed architecture makes a virtual area based on location information, which is collected via mobile networks, and then the nodes construct an ad hoc network via local networks. Fig. 1 shows the concept of the proposed architecture and an example of the virtual area using relay range restriction of ad hoc networks. Note that the proposed architecture is not carrier-assisted D2D. This method use a mobile network only for connecting to the global network as a network infrastructure since it does not need to rely on any information and to deploy any mechanism on carrier-assisted facilities. Namely, it realizes interoperable ad hoc networking over multiple mobile network carriers. The proposed architecture comprises the location layer and the ad hoc layer.

\section{(1) Location layer}

The roles of the location layer are to manage the locations of nodes and to determine the virtual area of each ad hoc network by a management server via mobile network. In our method, the server that manages the location information utilizes the server of the proposed method [5]. Note that, although an ad-hoc networking service provider (e.g. organization, user, etc.) has to deploy the server on the global network to collect and manage node's information, the provider does 
not have to be the same as mobile network carrier since the provider does not need to take any part in the physical network. In addition, the location layer never performs the actual route construction and any data transmissions, and hence the ad hoc layer must perform all of the actual routing via local networks as well as the traditional ad hoc networks.

First, each node periodically sends a location registration message including its address and location to the server via mobile networks. Then, the server holds the received information for a certain period. Based on the information, the server provides the location information of nodes within a virtual area.

When a source wants to communicate with another node via local networks, it sends a location request message including its address and destination address to the server via mobile network. In receiving the message, the location server determines the virtual area for ad hoc networking based on a relay range restriction as described in Sec. 2.2. If the server succeeds to determine the virtual area, it sends a message including the virtual area information to the source, and then the source begins the route construction process via local networks. If the server fails to determine the area, it notifies the failure to the source.

\section{(2) Ad hoc layer}

The roles of the ad hoc layer are to establish an actual route and then to send data along the established route via local networks.

After receiving the virtual area information from the server via mobile networks, the source initiates the route construction based on a routing protocol via

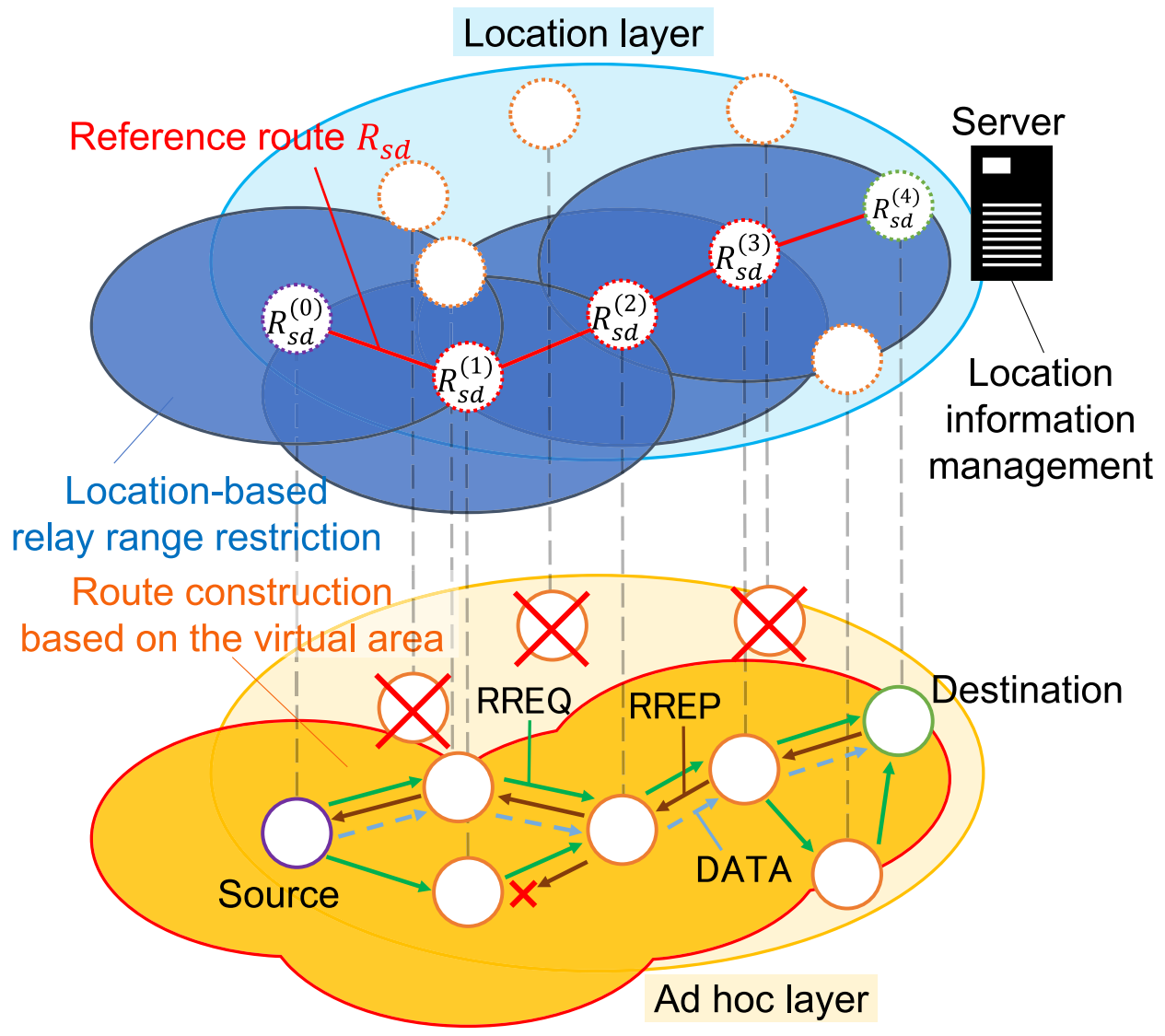

Fig. 1. Concept of the proposed architecture and example of the virtual area. As an example, the ad hoc layer adopts a traditional reactive routing protocol. 
local networks. After the route construction, the source starts the data transmission along the established route. Note that the server only informs the virtual area information for ad hoc networking to the source, and therefore it does not rely on a specific ad hoc routing protocol. The details of the route construction are described in Sec. 2.3.

\subsection{Relay range restriction mechanism based on location informa- tion}

This section introduces a relay range restriction mechanism to determine a virtual area to reduce the network load caused by the route discovery process. The proposed mechanism calculates a "reference route" to narrow the relay range to the neighbor area of the reference route. Note that each node within the reference route is called "reference node".

The details of the algorithm are shown in Alg. 1, and an example of the relay range restriction is shown in Fig. 1. Here, $h_{\max }$ denotes the maximum hop count, the function $d(i, j)$ denotes the distance between nodes $i$ and $j$, and the function $n(i)$ denotes the set of the $i$ 's neighbors within the reference communication range $C_{\max }$, respectively. $R_{s d}$ denotes the reference node set in the reference route between nodes $s$ and $d . R_{s d}$ is sorted based on the hop count in the order from $s$ to $d$, and hence $R_{s d}^{(i)}\left(0 \leq R_{s d}^{(i)} \leq h_{\max }\right)$ denotes the $i$-th hop node on the route.

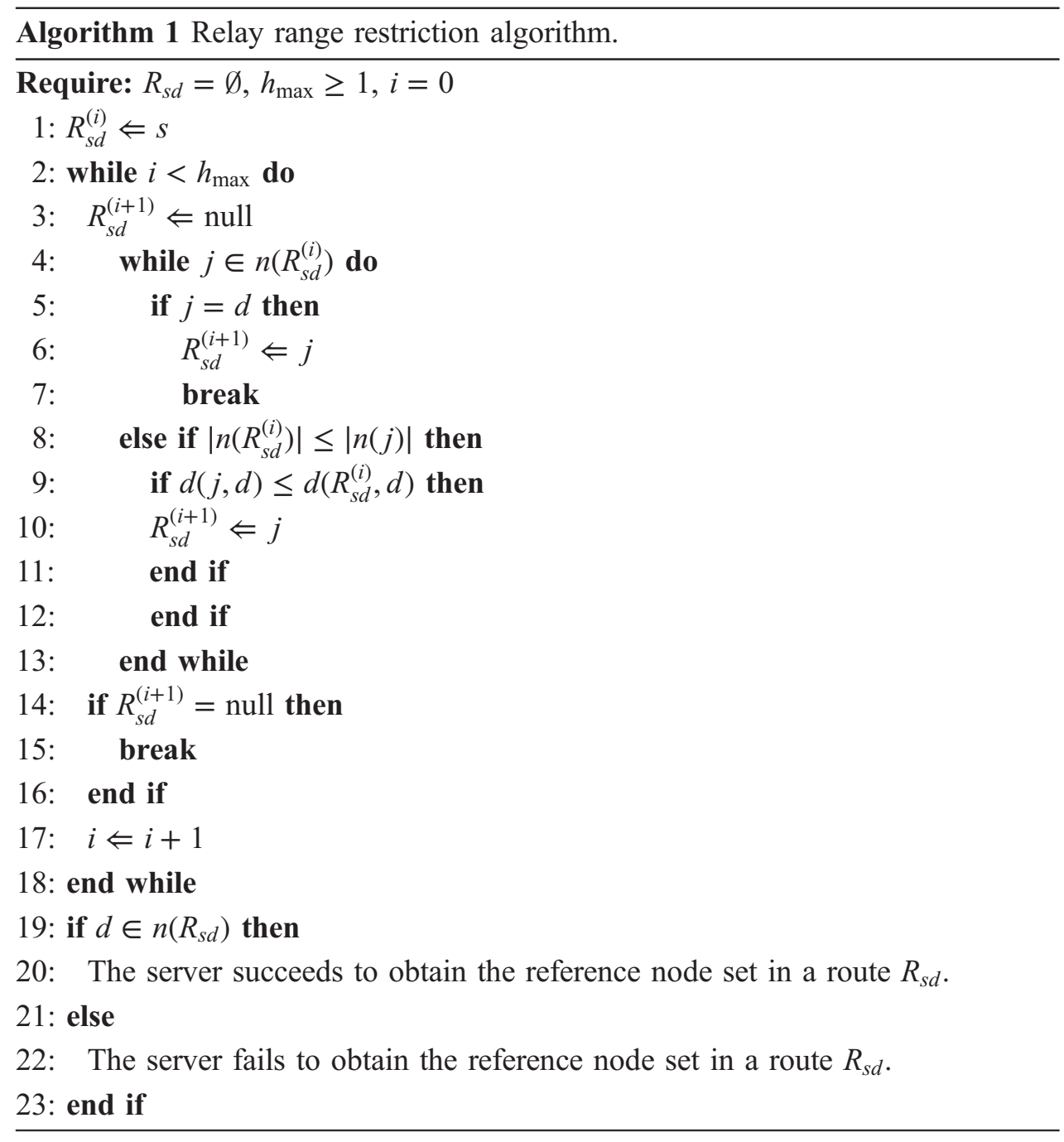


To calculate $R_{s d}$, the algorithm recursively searches the next-hop reference node $R_{s d}^{(i+1)}$ from $n(i)$. Here, $R_{s d}^{(i+1)}$ will be selected so that (1) $R_{s d}^{(i+1)}$ is closer to $d$ than $R_{s d}^{(i)}$, and (2) $R_{s d}^{(i+1)}$ has the largest number of neighbors among the elements of $n(i)$ to avoid sparse areas. If $R_{s d}^{(i+1)}$ coincides with $d$, the server determines the reference route $R_{s d}$. Here, the server makes the virtual area based on $C_{\max }$ of each node within $R_{s d}$ except for $d$. If all the elements of $n(i)$ do not satisfy the conditions or $i$ exceeds $h_{\max }$, it fails to determine a reference route.

\subsection{Routing mechanism based on virtual area}

This section introduces the routing mechanism via local networks based on a virtual area using the relay range restriction. As mentioned above, the routing mechanism does not depend on a specific routing protocol. To introduce the mechanism clearly, this letter adopts AODV [6].

After receiving the virtual area information from the server as described in Sec. 2.2, the source begins to flood the route request towards the destination. Here, the route request includes the virtual area information in addition to the original information of route request. The virtual area information consists of the reference route, locations of reference nodes, and the reference communication range $C_{\max }$. On the ad hoc layer, when a node receives the route request, it checks whether it exists in the virtual area. If the node exists in the area, it relays the route request; otherwise, it ignores and discards the route request. When the destination receives the route request, it sends the route reply towards the source using the reverse path of the route request traversed path. Finally, when the source receives the route reply, it begins the data transmission using the established route on the ad hoc layer.

\section{Performance evaluation}

\subsection{Simulation environment}

Our simulations evaluated the performance using ns3 [7]. The simulation area was set to $1000 \mathrm{~m} \times 1000 \mathrm{~m}$. IEEE $802.11 \mathrm{a}$ was used as the wireless medium, the communication range was set to about $100 \mathrm{~m}$, and the data rate was set to $6 \mathrm{Mbps}$. To evaluate the impact of the node density, this simulation varied the number of nodes from 100 to 500 . The number of source was set to 20. Each source sends a 1 Kbyte packet 20 times to randomly selected destination using UDP. In the proposed architecture, $C_{\max }$ was set to $100 \mathrm{~m}$, and $h_{\max }$ was set to 35 .

\subsection{Simulation results}

Total amount of control packets. Fig. 2(a) shows that AODV significantly increases the total amount of control messages as the node density increases due to the message flooding in dense environments. Here, "Proposed(local)" shows that amount of control messages without using mobile networks and "Proposed(local + mobile)" shows that total amount of control messages in our method. In addition, the proposed architecture significantly reduces them to restrict the relay range even if it periodically sends control messages via mobile networks.

Packet arrival rate. Fig. 2(b) shows that AODV degrades the packet arrival rate since the packet collisions occur due to the network-wide message flooding especially in dense environments. In contrast, the proposed architecture always 


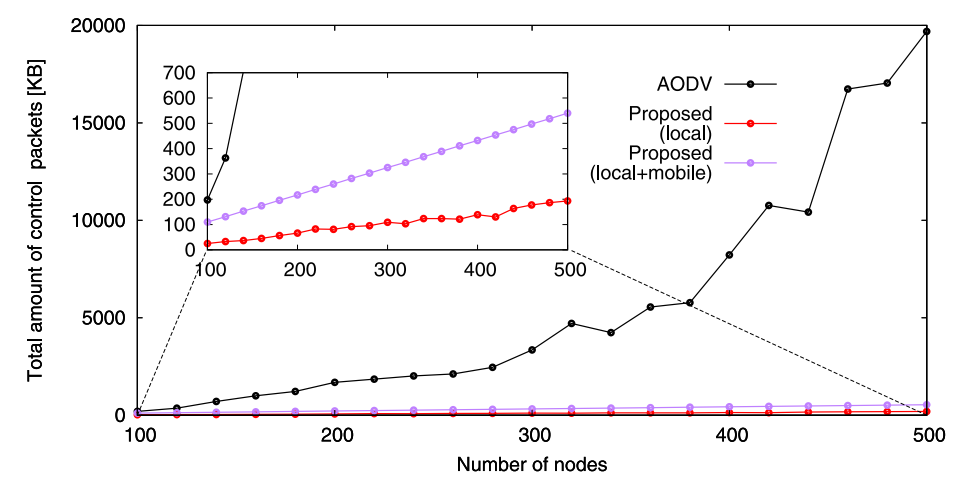

(a) Total amount of control messages.

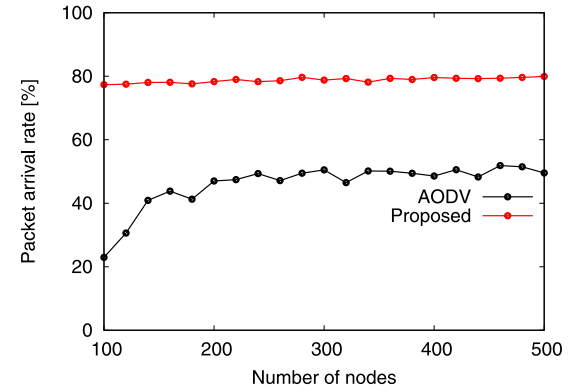

(b) Packet arrival rate.

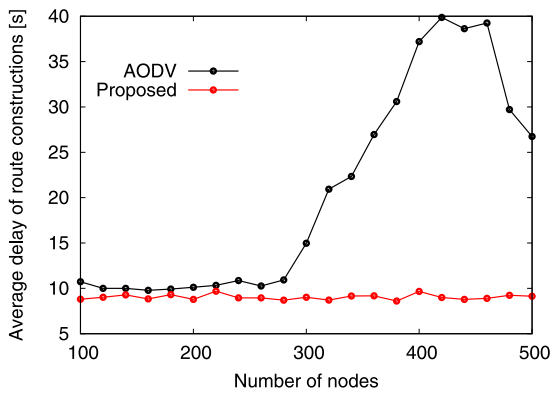

(c) Average delay of route constructions.

Fig. 2. Simulation results.

achieves the higher packet arrival rate than AODV since it reduces the network load by narrowing the relay range especially in dense environments.

Average delay of route constructions. Fig. 2(c) shows that the proposed architecture always achieves the lower delay of route constructions than AODV since it restricts the message flooding along the neighbor area of the reference route and thus achieves the reduction of unnecessary control messages.

From the results, the proposed architecture achieves the reductions of unnecessary control messages and route construction delay, and the improvement of the packet arrival rate.

\section{Conclusions}

This letter proposed a mobile-assisted ad hoc networking architecture based on location information. The proposed architecture realized the efficient routing mechanism with the location-based relay range restriction.

From the simulation results, by reducing the unnecessary control messages by narrowing the relay range, the proposed architecture achieves the improvement of the packet arrival rate and the reductions of network load and delay.

Currently, the simulations do not consider the packet loss in mobile networks. Hence, we should consider that for evaluating the performance in realistic environments. In addition, the relay range restriction does not consider the variation of the communication range. In real environments, the communication range of each node varies, and hence the estimation mechanism of them is necessary.

\section{Acknowledgments}

This work was supported by JSPS KAKENHI Grant Number 17K12680. 